\title{
Spatial behaviour in thermoelastostatic cylinders of indefinitely increasing cross-section
}

\author{
R. J. Knops \\ The Maxwell Institute of Mathematical Sciences \\ Heriot-Watt University, \\ Edinburgh, Scotland, U.K. \\ R Quintanilla* \\ Matemática Aplicada 2,UPC \\ C.Colón 11, 08222 Terrassa, \\ Barcelona, Spain
}

February 25, 2015

\begin{abstract}
Alternative growth and decay estimates, reminiscent of the classical Phragmén-Lindelöf principle, are derived for a linearised thermoelastic body whose plane cross-sections increase unboundedly with respect to a given direction. The proof uses a modified Poincaré inequality to construct a differential inequality for a weighted linear combination of the cross-sectional mechanical and thermal energy fluxes. Decay estimates are deduced also for the cross-sectional mean square measures of the displacement and temperature. An explicit upper bound in terms of base data is established for the amplitude occurring in the decay estimates.
\end{abstract}

Keywords: Thermoelastostatics; increasing cross-sections; Phragmén-Lindelöf principle.

\section{Introduction}

Spatial stability in equilibrium theories of elasticiy and thermoelasticity may be established from estimates for spatial (average) behaviour, usually measured by the energy, in terms of distance from the load surface. Such studies, clearly related to Saint-Venant's principle, are significant for investigations into boundary effects and similar phenomena. In elasticity, both growth and decay estimates have been derived for nonprismatic and prismatic finite and semi-infinite

* Supported by the Project "Anáisis Matemático de las Ecuaciones en Derivada Parciales del Termomecánica “(MTM2013-42004-P) of the Spanish Ministry of Economy and Competitiveness. 
cylinders (e.g., [1, 2]), and for cone-like regions (e.g., [5]) unbounded in a given direction. The method of derivation, however, does not readily extend to thermoelasticity, although the cylinder has been successfully treated by separate weighting of the mechanical and thermal energies [6]. The weight function, however, becomes unbounded when the cylinder's cross-section increases unboundedly, and consequently is inappropriate for problems considered in this paper.

We treat a linearised classical thermoelastic body that becomes unbounded in at least one direction $x_{1}$ and whose plane cross-sections perpendicular to this direction are contained between a divergent wedge. Consequently, they are of indefinitely increasing area. A cone is included, but equally the crosssections may extend to infinity in directions that, for example, are parallel to the intersection of the wedge and cross-sections. For simplicity, it is supposed that the displacement and temperature are specified non-zero on the base $x_{1}=0$, but are homogeneous on the lateral sides. Asymptotic behaviour as $x_{1}$ tends to infinity is not, however, prescribed. Constitutive coefficients satisfy physically plausible restrictions that include positive-definiteness and upper boundedness. The sum of the mechanical and weighted heat energy fluxes across each plane cross-section, where the polynomial weight function depends upon the single variable $x_{1}$, is shown to satisfy a first order differential inequality which on integration establishes conditions for the growth and decay of the solution with respect to $x_{1}$, similar to the Phragmén-Lindelöf principle in potential theory. The estimates, for which the solution is measured either by the weighted sum of cross-sectional energy fluxes or volume energies, assume the forms respectively of an exponentially increasing lower bound, or an exponentially decreasing upper bound. Under conditions for decay, the mean square cross-sectional measures of both the displacement and temperature satisfy similar decay estimates.

The calculations rely upon three inequalities, the first of which corresponds to a modified Poincaré inequality and is derived in Section 2, where geometric properties are also described. Section 3 defines the boundary value problem to be studied and presents the assumptions adopted for the various constitutive coefficients. The remaining two principal inequalities, constructed in Section 4, are expressed in terms of a certain function that involves the temperature and its spatial derivatives. The inequalities are used in Section 5 to obtain a first order differential inequality for a weighted linear combination of the mechanical and thermal energy fluxes. In Section 6, reference to results discussed in [2], where a similar differential inequality is obtained, easily leads to the present alternative exponential growth and decay estimates corresponding to the PhragménLindelöf principle. Optimal decay rates are determined in Section 6.1. Section 7 develops procedures for the asymptotic decay of the displacement and temperature in terms of their respective mean-square cross-sectional measures, while Section 8 examines implications for growth. The amplitude in the decay estimates lacks physical interest since its dependence on base data is not explicit. The defect is remedied in Section 9 where a bound for the amplitude is obtained specifically in terms of the base data. Section 10 concludes the main part of the paper. It briefly examines the uncoupled mechanical and thermal problems and 
derives the corresponding estimeated growth and decay rates by the method of this paper. The mechanical and thermal rates are simply compared not only with each other, but also with those obtained by application of the method simultaneously to both uncoupled problems. Expressions for the respective rates in the coupled problem are somewhat complex preventing easy comparision. An Appendix is devoted to several methods for selecting the arbitrary constants occurring in the previous calculations in order to derive explicit decay and growth rates. These rates are not, however, optimal.

The standard comma notation is employed to indicate partial differentiation, and the summation convention is applied, with Roman subscripts in the range $1,2,3$. Greek subscripts are restricted mainly to 2 and 3. An indicial notation is also adopted to represent components of vectors and tensors which are taken with respect to the same Cartesian coordinate system unless stated otherwise. Existence of a suitably differentiable solution is assumed throughout.

\section{Preliminaries}

Consider a Cartesian system of rectangular coordinates $x_{1} x_{2} x_{3}$ and let the spatial region $\Omega \subseteq\left\{x \in \mathbb{R}^{3}: x_{1} \geq 0\right\}$ be an unbounded non-prismatic cylinder that extends to infinity as $x_{1} \rightarrow \infty$. We introduce the notation

$$
\Omega(z)=\left\{x \in \Omega, x_{1} \geq z\right\},
$$

and denote a plane cross-section of the cylinder by

$$
D(z)=\left\{x \in \Omega, x_{1}=z\right\} .
$$

The boundary $\partial D(z)$ of $D(z)$ is supposed sufficiently smooth to admit applications of the divergence theorem. We let $D(z)$ become unbounded as $z \rightarrow \infty$ in either of the following ways:

(I) The cross-section $D(z)$ is contained between two parallel lines distance $l(z)$ apart for every $z \geq 0$, where

$$
l(z) \leq \pi C(z+h)^{\alpha}, \quad C>0, h>0, \alpha \in(0,1],
$$

and the positive constants $C, h, \alpha$ are chosen to satisfy the relation

$$
1-\frac{2 C \alpha \tilde{k}^{1 / 2}}{h^{1-\alpha} k_{1}^{1 / 2}}=C_{1}>0
$$

in which $k_{1}, \tilde{k}$ are prescribed constants related to the heat conduction tensor by (3.8) and $(3.10)_{2}$.

When $\alpha=1$, condition (2.4) is replaced by

$$
1-\frac{2 C \tilde{k}^{1 / 2}}{k_{1}^{1 / 2}}=C_{1}>0
$$

which is satisfied for all $\tilde{k}, k_{1}$ by suitable choice of the constant $C$. 
(II) The area $|D(z)|$ of the cross-section $D(z)$ satisfies the upper bound

$$
|D(z)| \leq \pi j_{0}^{2} C^{2}(z+h)^{2 \alpha}, C>0, h>0, \alpha \in(0,1],
$$

where $C, h, \alpha$ satisfy condition (2.4), or $C$ satisfies (2.5) when $\alpha=1$, and $j_{0}$ is the first zero of the Bessel function of order zero $\left(j_{0} \sim 2.408 \ldots\right)$.

Regions in Category I are more general than those in Category II since crosssections with $z<\infty$ are allowed to become unbounded in directions parallel to that of the given lines. For this reason, the solution must belong to the function space $W^{1,2}(D(z)), z \geq 0$, or more generally $W^{1,2}(\Omega)$. Otherwise, we suppose that $D(z)$ remains bounded for all finite $z$.

The following provide examples of spatial regions in each category:

Category 1. Suppose $\Omega=\left\{x, x_{1} \geq 0,\left|x_{2}\right| \leq \pi C\left(x_{1}+h\right)^{\alpha},\left|x_{3}\right| \leq C^{0}\left(x_{1}+\right.\right.$ $\left.h)^{\alpha}\right\}$ where $C>0, C^{0}>0, h>0$ are constants, and $\alpha \in(0,1]$. In this example $D(0)=\left\{x, x_{1}=0,\left|x_{2}\right| \leq \pi C h^{\alpha},\left|x_{3}\right| \leq C^{0} h^{\alpha}\right\}$ and $D(z)=\left\{x, x_{1}=z,\left|x_{2}\right| \leq\right.$ $\left.\pi C(z+h)^{\alpha},\left|x_{3}\right| \leq C^{0}(z+h)^{\alpha}\right\}$.

Category 2. Suppose $\Omega=\left\{x, x_{1} \geq 0, x_{2}^{2}+x_{3}^{2} \leq \pi j_{0}^{2} C^{2}\left(x_{1}+h\right)^{2 \alpha}\right\}$ where $C>0, h>0$ are constants and $\alpha \in(0,1]$. In this case, $D(0)=\left\{x, x_{1}=\right.$ $\left.0, x_{2}^{2}+x_{3}^{2} \leq \pi j_{0}^{2} C^{2} h^{2 \alpha}\right\}$ and $D(z)=\left\{x, x_{1}=z \geq 0, x_{2}^{2}+x_{3}^{2} \leq \pi j_{0}^{2} C^{2}(z+h)^{2 \alpha}\right\}$.

Figures 1 and 2 show examples of regions in Category I and II. Figure 1 depicts a wedge with curvilinear sides for which the parameters are chosen to be $C=h=1$ and $\alpha=1 / 4$.

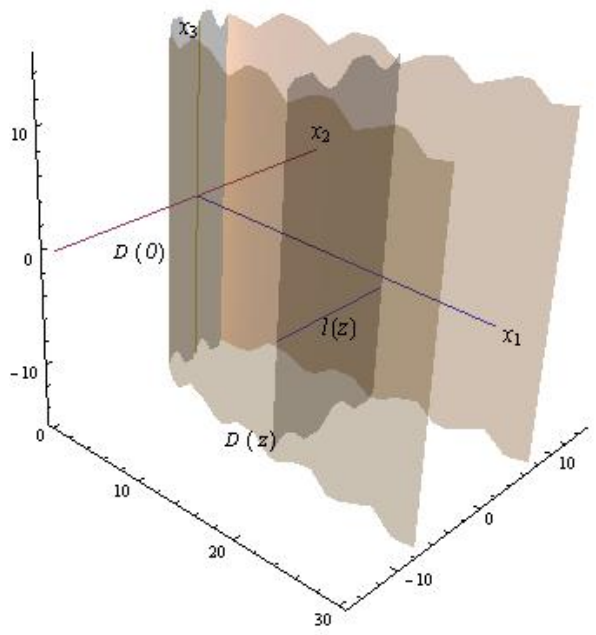

Figure 1: A Category I region

Figure 2 is an example of a cone-like region belonging to Category II whose parameters have the same value as those in Figure 1.

A modified Poincaré inequality, key to subsequent arguments, is established next. We have 


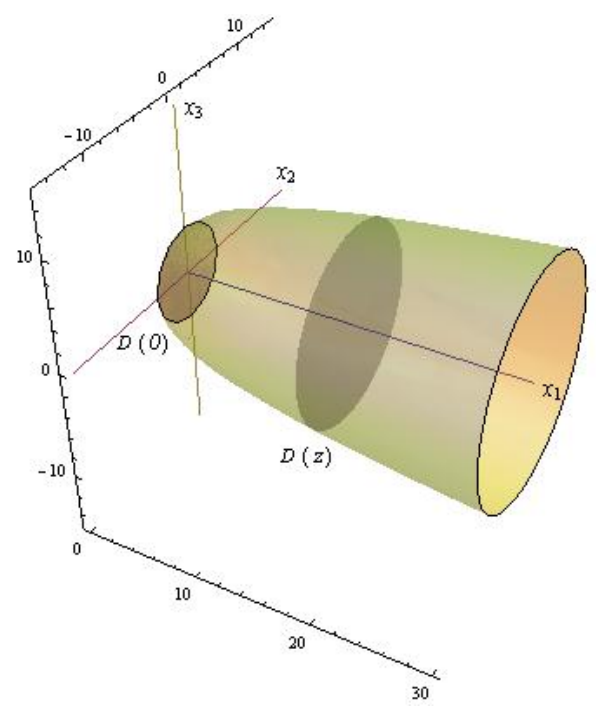

Figure 2: A Category II region

Lemma 2.1 Let the function $v \in W_{0}^{1,2}(D(z))$ be defined on $D(z)$ and satisfy the boundary condition

$$
v\left(x_{2}, x_{3}\right)=0, \quad\left(x_{2}, x_{3}\right) \in \partial D(z) .
$$

Then

$$
\int_{D(z)} v^{2} d a \leq C^{2}(z+h)^{2 \alpha} \int_{D(z)} v_{, \gamma} v_{, \gamma} d a, \quad \gamma=2,3
$$

holds for every $z \geq 0$, where da represents the element of area on $D(z)$.

Proof. For regions $D(z)$ belonging to Category II, inequality (2.8) follows from the standard Poincaré inequality

$$
\lambda(z) \int_{D(z)} v^{2} d a \leq \int_{D(z)} v_{, \alpha} v_{, \alpha} d a
$$

in conjunction with the Faber-Krahn inequality

$$
\lambda(z) \geq \frac{\pi j_{0}^{2}}{|D(z)|} .
$$

For Category I regions, without loss we may suppose the $x_{3}$ axis to be parallel to the given pair of parallel lines distance $l(z)$ apart, and consider the point $\left(x_{2}, \bar{x}_{3}\right) \in D(z)$ for fixed $\bar{x}_{3}$ and fixed $z \geq 0$. Let the straight line through $x_{2}, \bar{x}_{3}$ parallel to the $x_{2}$-axis have length $l\left(\bar{x}_{3}, z\right)$ and intercept the boundary 
$\partial D(z)$ in points $x_{2}^{0}\left(\bar{x}_{3}\right) \leq x_{2}^{1}\left(\bar{x}_{3}\right)$. The one- dimensional Wirtinger inequality given by (see, for example, [4, pp., 184-185])

$$
\int_{x_{2}^{0}\left(\bar{x}_{3}\right)}^{x_{2}^{1}\left(\bar{x}_{3}\right)} v^{2}\left(\xi, \bar{x}_{3}\right) d \xi \leq \frac{l^{2}\left(\bar{x}_{3}, z\right)}{\pi^{2}} \int_{x_{2}^{0}\left(\bar{x}_{3}\right)}^{x_{2}^{1}\left(\bar{x}_{3}\right)}\left|v_{, \xi}\left(\xi, \bar{x}_{3}\right)\right|^{2} d \xi,
$$

immediately yields

$$
\begin{aligned}
\int_{x_{2}^{0}\left(\bar{x}_{3}\right)}^{x_{2}^{1}\left(\bar{x}_{3}\right)} v^{2}\left(\xi, \bar{x}_{3}\right) d \xi & \leq \frac{l^{2}(z)}{\pi^{2}} \int_{x_{2}^{0}\left(\bar{x}_{3}\right)}^{x_{2}^{1}\left(\bar{x}_{3}\right)}\left|v_{, \xi}\left(\xi, \bar{x}_{3}\right)\right|^{2} d \xi \\
& \leq C^{2}(z+h)^{2 \alpha} \int_{x_{2}^{0}\left(\bar{x}_{3}\right)}^{x_{2}^{1}\left(\bar{x}_{3}\right)}\left|v_{, \xi}\left(\xi, \bar{x}_{3}\right)\right|^{2} d \xi
\end{aligned}
$$

by virtue of assumption (2.3). We now allow $\bar{x}_{3}$ to become arbitrary and integrate with respect to this variable over its extreme values in $D(z)$ to obtain the stated inequality $(2.8)$.

\section{Linearised thermoelastostatics}

In the absence of supply terms, the equilibrium equations of linearised compressible inhomogenous thermoelasticity for the stress $t_{i j}$ and heat flux $q_{i}$ become

$$
\begin{aligned}
t_{i j, j} & =0, & & x \in \Omega, \\
q_{i, i} & =0, & & x \in \Omega,
\end{aligned}
$$

where

$$
\begin{aligned}
t_{i j} & =d_{i j k l} u_{k, l}+\beta_{i j} \theta, \quad x \in \Omega, \\
q_{i} & =k_{i j} \theta_{, j}, \quad x \in \Omega .
\end{aligned}
$$

Here, $u_{i}(x)$ are the Cartesian components of the displacement vector, $\theta(x)>0$ is the temperature, $\beta_{i j}(x)$ are the Cartesian components of the symmetric thermal coupling tensor, $k_{i j}(x)$ are the Cartesian components of the symmetric heat conduction tensor, and $d_{i j k l}(x)$ are the Cartesian components of the elasticity tensor that possess only the major symmetry

$$
d_{i j k l}=d_{k l i j} .
$$

In general, the kinematical and thermal constitutive functions are differentiable with respect to position $x_{i}$.

Substitution of the constitutive relations (3.3) and (3.4) in the equilibrium equations (3.1) and (3.2) yields

$$
\begin{array}{rlrl}
\left(d_{i j k l} u_{k, l}+\beta_{i j} \theta\right)_{, j} & =0, & & x \in \Omega, \\
\left(k_{i l} \theta_{, l}\right)_{, i} & =0, & x \in \Omega .
\end{array}
$$

The following conditions are assumed for the constitutive functions: 
(i) The heat conduction tensor is positive definite and posssesses an upper bound in the sense that positive constant $k_{1}$ and $\bar{k}$ exist such that

$$
\begin{aligned}
& k_{i j} \xi_{i} \xi_{j} \geq k_{1} \xi_{i} \xi_{i}, \quad \text { for every vector } \xi_{i}, \\
& k_{i j} \xi_{i} \xi_{j} \leq \bar{k} \xi_{i} \xi_{i}, \quad \text { for every vector } \xi_{i} .
\end{aligned}
$$

(ii) The coupling tensor $\beta_{i j}$ and the function $k_{11}$ possess upper bounds respectively denoted by

$$
\widetilde{\beta}=\sup _{\Omega} \beta_{i j} \beta_{i j}, \quad \widetilde{k}=\sup _{\Omega} k_{11},
$$

(iii) Similarly, the elasticity tensor $d_{i j k h}$ is positive definite and bounded above in the sense positive constants $d_{1}$ and $\widetilde{d}$ exist such that

$$
\begin{aligned}
d_{i j k h} \xi_{i j} \xi_{k h} & \geq d_{1} \xi_{i j} \xi_{i j}, \quad \text { for every tensor } \xi_{i j}, \\
d_{i j k h} \xi_{i j} \xi_{k h} & \leq \widetilde{d} \xi_{i j} \xi_{i j}, \quad \text { for every tensor } \xi_{i j} .
\end{aligned}
$$

These assumptions are physically plausible. Condition (3.8) is compatible with the second law of thermodynamics that requires the heat conduction tensor to be non-negative. A positive-definite elasticity tensor is related to the stability of the solutions with respect to small elastodynamic perturbations.

To complete the specification of the boundary value problem (3.6) and (3.7) we adjoin Dirichlet boundary conditions prescribed by

$$
u_{i}=\theta=0, \quad x \in \partial D(z), \quad z \geq 0,
$$

and

$$
u_{i}\left(0, x_{2}, x_{3}\right)=f_{i}\left(x_{2}, x_{3}\right), \quad \theta\left(0, x_{2}, x_{3}\right)=g\left(x_{2}, x_{3}\right), \quad\left(0, x_{2}, x_{3}\right) \in D(0),
$$

where $f_{i}, g$ are prescribed functions.

Compatibility of these boundary conditions is ensured by the assumption that

$$
f_{i}\left(x_{2}, x_{3}\right)=g\left(x_{2}, x_{3}\right)=0, \quad\left(x_{2}, x_{3}\right) \in \partial D(0) .
$$

Dirichlet boundary conditions are chosen for simplicity, but other standard types can be included in the analysis subject to suitable normalisations for those of Neumann type.

Before establishing spatial behaviour of the solution to our boundary value problem, we derive some additional inequalities. It is also convenient to introduce the following further notation

$$
\begin{gathered}
d=\left(\frac{\widetilde{d}}{d_{1}}\right)^{1 / 2}, \quad k=\left(\frac{\widetilde{k}}{k_{1}}\right)^{1 / 2}, \\
a=\left(\frac{\widetilde{\beta}}{d_{1} k_{1}}\right)^{1 / 2}, \quad b=\frac{a C}{2 C_{1}^{1 / 2}}, \quad e=\frac{2 \alpha}{h^{1-\alpha}} .
\end{gathered}
$$


In this notation, condition (2.4) becomes

$$
C_{1}=1-k e C>0
$$

where

$$
0<k<(e C)^{-1} .
$$

which for assigned $k$ always hold for sufficiently small $e C$, as noted in (2.4). A similar remark applies to (2.5).

\section{Inequalities}

The main results of the paper also rely upon inequalities which are stated and proved in the following lemmas both of which involve the function

$$
G(z)=\Lambda(z) \int_{D(z)} k_{i j} \theta_{, i} \theta_{, j} d a+\Lambda^{\prime}(z) \int_{D(z)} k_{i 1} \theta_{, i} \theta d a,
$$

where

$$
\Lambda(z)=\lambda(z+h)^{2 \alpha}, \quad \lambda>0, \quad h>0, \quad \alpha \in(0,1],
$$

and the positive constant $\lambda$ is to be chosen. A superposed prime indicates differentiation with respect to the argument.

Lemma 1. Assume that $\Omega$ belongs to either Category I or II, and that the respective conditions (2.4) or (2.5) hold. Then

$$
G(z) \geq C_{1} \Lambda(z) \int_{D(z)} k_{i j} \theta_{, i} \theta_{, j} d a .
$$

Proof. By hypothesis, we have $\Lambda^{\prime}(z) \geq 0$ for every $z \geq 0$. Note also that the unit normal on $D(z)$ in the positive $x_{1}$-direction is given by $n=(1,0,0)$. Then by Schwarz's inequality and condition (3.8) we obtain

$$
\begin{aligned}
\left|\Lambda^{\prime}(z) \int_{D(z)} k_{i 1} \theta_{, i} \theta d a\right| & =\left|\Lambda^{\prime}(z) \int_{D(z)} k_{i j} \theta_{, i} n_{j} \theta d a\right| \\
& \leq \Lambda^{\prime}(z)\left[\int_{D(z)} k_{i j} \theta_{, i} \theta_{, j} d a \int_{D(z)} k_{i j} n_{i} n_{j} \theta^{2} d a\right]^{1 / 2} \\
& =\Lambda^{\prime}(z)\left[\int_{D(z)} k_{i j} \theta_{, i} \theta_{, j} d a \int_{D(z)} k_{11} \theta^{2} d a\right]^{1 / 2} \\
& \leq C(z+h)^{\alpha} k \Lambda^{\prime}(z) \int_{D(z)} k_{i j} \theta_{, i} \theta_{, j} d a,
\end{aligned}
$$

where $k$ is defined in $(3.16)_{2}$, and inequality (2.8) together with definition $(3.10)_{2}$ are additionally used. After insertion of (4.4) into (4.1) and upon noting that 


$$
\begin{aligned}
& (z+h)^{(\alpha-1)} \leq h^{(\alpha-1)} \text { for } 0<\alpha \leq 1 \text { and } z \geq 0, \text { we obtain } \\
& \qquad \begin{aligned}
G(z) & \geq\left[\Lambda(z)-2 \alpha C \lambda k(z+h)^{3 \alpha-1}\right] \int_{D(z)} k_{i j} \theta_{, i} \theta_{, j} d a \\
& \geq \Lambda(z)\left[1-2 \alpha C k h^{\alpha-1}\right] \int_{D(z)} k_{i j} \theta_{, i} \theta_{, j} d a .
\end{aligned}
\end{aligned}
$$

The lemma is established on recalling conditions (2.4) and (2.5) .

Remark 4.1 It easily follows from (2.8) and (4.3) that

$$
\begin{aligned}
\int_{D(z)} \theta^{2} d a & \leq C^{2}(z+h)^{2 \alpha} \int_{D(z)} \theta_{, i} \theta_{, i} d a \\
& \leq \frac{C^{2} \Lambda(z)}{\lambda k_{1}} \int_{D(z)} k_{i j} \theta_{, i} \theta_{, j} d a \\
& \leq \frac{C^{2}}{\lambda C_{1} k_{1}} G(z), \quad z \geq 0 .
\end{aligned}
$$

Lemma 2. Let $\Omega$ belong to Category I or II and suppose either condition (2.4) or condition (2.5) hold. Then, there exists a positive constant $\gamma_{1}$ such that

$$
\left|\int_{D(z)} \beta_{i j} u_{i, j} \theta d a\right| \leq \frac{a C}{2}\left[\gamma_{1}^{-1} \int_{D(z)} d_{i j k h} u_{i, j} u_{k, h} d a+\gamma_{1}(z+h)^{2 \alpha} \int_{D(z)} k_{i j} \theta_{, i} \theta_{, j} d a\right]
$$

where $a$ is given by $(3.17)_{1}$.

Proof. Schwarz's inequality, inequality (2.8), assumptions (3.8), (3.11), and $(3.10)_{1}$ together with (4.6) lead to

$$
\begin{gathered}
\left|\int_{D(z)} \beta_{i j} u_{i, j} \theta d a\right| \leq\left(\int_{D(z)} \beta_{i j} \beta_{i j} \theta^{2} d a \int_{D(z)} u_{i, j} u_{i, j} d a\right)^{1 / 2} \\
\leq a C(z+h)^{\alpha}\left(\int_{D(z)} k_{i j} \theta_{, i} \theta_{, j} d a \int_{D(z)} d_{i j k h} u_{i, j} u_{k, h} d a\right)^{1 / 2} \\
\leq \frac{a C(z+h)^{\alpha}}{2}\left(\epsilon(z) \int_{D(z)} k_{i j} \theta_{, i} \theta_{, j} d a+\epsilon^{-1}(z) \int_{D(z)} d_{i j k h} u_{i, j} u_{k, h} d a\right) .
\end{gathered}
$$

where the arithmetic-geometric mean inequality is used for the last inequality and $\epsilon(z)$ is an arbitrary positive function depending upon the parameter $z \geq 0$. On setting

$$
\epsilon(z)=\gamma_{1}(z+h)^{\alpha}
$$

we obtain inequality (4.8). 


\section{Differential inequality}

Preparatory to a Phragmén-Lindelöf alternative established in the next section, we construct in this section a differential inequality for solutions to the boundary value problem specified by the system (3.6) and (3.7) and the boundary conditions (3.13) and (3.14). Consider the function

$$
F(z)=\int_{D(z)}\left(d_{i 1 k h} u_{k, h}+\beta_{i 1} \theta\right) u_{i} d a+\mu \Lambda(z) \int_{D(z)} k_{i 1} \theta_{, i} \theta d a,
$$

where $\Lambda(z)$ is the function defined by (4.2), and $\mu$ is a positive constant to be chosen.

Let $s \geq 0$. Integration by parts gives

$$
F(z+s)-F(z)=\int_{z}^{z+s} \int_{D(\eta)}\left(d_{i j k h} u_{i, j} u_{k, h}+\beta_{i j} u_{i, j} \theta\right) d a d \eta+\mu \int_{z}^{z+s} G(\eta) d \eta
$$

where $G(z)$ is the function defined by (4.1). It follows that

$$
F^{\prime}(z)=\int_{D(z)}\left(d_{i j k h} u_{i, j} u_{k, h}+\beta_{i j} u_{i, j} \theta\right) d a+\mu G(z) .
$$

Employment of Lemmas 1 and 2 in the last expression gives the inequality

$$
\begin{aligned}
F^{\prime}(z) \geq & \left(1-\frac{a C}{2 \gamma_{1}}\right) \int_{D(z)} d_{i j k l} u_{i, j} u_{k, l} d a \\
& +\left(\mu C_{1}-\frac{a C \gamma_{1}}{2 \lambda}\right) \Lambda(z) \int_{D(z)} k_{i j} \theta_{, i} \theta_{, j} d a \\
= & A \int_{D(z)} d_{i j k l} u_{i, j} u_{k, l} d a+B \Lambda(z) \int_{D(z)} k_{i j} \theta_{, i} \theta_{, j} d a \\
= & A\left[\int_{D(z)} d_{i j k l} u_{i, j} u_{k, l} d a+A^{-1} B \Lambda(z) \int_{D(z)} k_{i j} \theta_{, i} \theta_{, j} d a\right]
\end{aligned}
$$

where

$$
\begin{aligned}
& A=\left(1-\frac{a C}{2 \gamma_{1}}\right), \\
& B=\left(\mu C_{1}-\frac{a C \gamma_{1}}{2 \lambda}\right) .
\end{aligned}
$$

The coefficients $A$ and $B$ are strictly positive provided $\gamma_{1}$ satisfies

$$
\frac{a C}{2}<\gamma_{1}<\frac{2 \lambda \mu C_{1}}{a C},
$$

which holds provided $\lambda$ and $\mu$ are chosen such that

$$
\left(\frac{a C}{2}\right)^{2}<\lambda \mu C_{1}
$$


In what follows, conditions (5.8) and (5.9) are always assumed.

A first order differential inequality satisfied by $F(z)$ is next derived by estimating the absolute value of the function $F(z)$ in terms of its derivative. It is immediate from expression (5.1) that

$$
|F(z)| \leq\left|I_{1}(z)\right|+\left|I_{2}(z)\right|+\left|I_{3}(z)\right|,
$$

where

$$
\begin{aligned}
& I_{1}(z)=\int_{D(z)} d_{i 1 k h} u_{k, h} u_{i} d a, \\
& I_{2}(z)=\int_{D(z)} \beta_{i 1} \theta u_{i} d a
\end{aligned}
$$

and

$$
I_{3}(z)=\mu \Lambda(z) \int_{D(z)} k_{i 1} \theta_{, i} \theta d a .
$$

Arguments similar to those used in Section 4 enable each of the above terms to be separately bounded. We have

$$
\begin{aligned}
& \left|I_{1}(z)\right| \leq\left(\int_{D(z)} d_{i j k h} u_{i, j} u_{k, h} d a \int_{D(z)} d_{i j k h} u_{i} n_{j} u_{k} n_{h} d a\right)^{1 / 2} \\
& \leq \widetilde{d}^{1 / 2}\left(\int_{D(z)} d_{i j k h} u_{i, j} u_{k, h} d a \int_{D(z)} u_{i} u_{i} d a\right)^{1 / 2} \\
& \leq d C(z+h)^{\alpha} \int_{D(z)} d_{i j k h} u_{i, j} u_{k, h} d a \\
& \left|I_{2}(z)\right| \leq\left(\int_{D(z)} u_{i} u_{i} d a \int_{D(z)} \beta_{i j} \beta_{i j} \theta^{2} d a\right)^{1 / 2} \\
& \leq \widetilde{\beta}^{1 / 2}\left(\int_{D(z)} u_{i} u_{i} d a \int_{D(z)} \theta^{2} d a\right)^{1 / 2} \\
& \leq a C^{2}(z+h)^{2 \alpha}\left(\int_{D(z)} d_{i j k h} u_{i, j} u_{k, h} d a \int_{D(z)} k_{i j} \theta_{, i} \theta_{, j} d a\right)^{1 / 2} \\
& \leq a C^{2}(z+h)^{\alpha}\left(\frac{1}{2 \gamma_{2}} \int_{D(z)} d_{i j k h} u_{i, j} u_{k, h} d a+\frac{\gamma_{2}}{2 \lambda} \Lambda(z) \int_{D(z)} k_{i j} \theta_{, i} \theta_{, j} d a\right), \\
& \left|I_{3}(z)\right| \leq \mu \Lambda(z)\left(\int_{D(z)} k_{i j} \theta_{, i} \theta_{, j} d a \int_{D(z)} k_{11} \theta^{2} d a\right)^{1 / 2} \\
& \leq \widetilde{k}^{1 / 2} \mu \Lambda(z)\left(\int_{D(z)} k_{i j} \theta_{, i} \theta_{, j} d a \int_{D(z)} \theta^{2} d a\right)^{1 / 2} \\
& \leq k C(z+h)^{\alpha} \mu \Lambda(z) \int_{D(z)} k_{i j} \theta_{, i} \theta_{, j} d a,
\end{aligned}
$$


where $\gamma_{2}$ is an arbitrary positive constant to be selected, and the constants $k$, $a$ are defined in terms of data by $(3.16)_{2}$ and $(3.17)_{1}$.

Substitution in (5.10) gives

$$
\begin{aligned}
|F(z)| \leq & C(z+h)^{\alpha}\left[\left(d+\frac{a C}{2 \gamma_{2}}\right) \int_{D(z)} d_{i j k l} u_{i, j} u_{k, l} d a\right. \\
& \left.+\left(\mu k+\frac{a C \gamma_{2}}{2 \lambda}\right) \Lambda(z) \int_{D(z)} k_{i j} \theta_{, i} \theta_{, j} d a\right] \\
= & C(z+h)^{\alpha}\left[U \int_{D(z)} d_{i j k l} u_{i, j} u_{k, l} d a\right. \\
& \left.+V \Lambda(z) \int_{D(z)} k_{i j} \theta_{, i} \theta_{, j} d a\right] \\
= & U C(z+h)^{\alpha}\left[\int_{D(z)} d_{i j k l} u_{i, j} u_{k, l} d a\right. \\
& \left.+U^{-1} V \Lambda(z) \int_{D(z)} k_{i j} \theta_{, i} \theta_{, j} d a\right],
\end{aligned}
$$

where

$$
\begin{aligned}
U & =\left(d+\frac{a C}{2 \gamma_{2}}\right), \\
V & =\left(\mu k+\frac{a C \gamma_{2}}{2 \lambda}\right) .
\end{aligned}
$$

Now suppose that the constants $\gamma_{1}$ and $\gamma_{2}$ are selected to satisfy

$$
A V \leq B U .
$$

Then (5.5) and (5.12) imply that

$$
|F(z)| \leq C^{*} C(z+h)^{\alpha} F^{\prime}(z),
$$

where $C^{*}=A^{-1} U$ is a positive constant dependent upon specific choices of $\gamma_{1}, \gamma_{2}$ described later. Meanwhile, we discuss the integration of (5.16).

\section{Phragmén-Lindelöf alternative}

Integration of the differential inequality (5.16) leads to an extension of the Phragmén-Lindelöf principle for alternative behaviour, familiar in classical potential theory. We refer to the procedure developed in [2] for the proof of the following result: 
Theorem 1. Assume that $\left(u_{i}, \theta\right)$ is a solution to the problem specified by the system (3.6) and (3.7) subject to boundary conditions (3.13) and (3.14). Then the function $F(z)$ defined by (5.1) alternatively satisfies the following:

Either

(a) there exists $z_{0} \geq 0$ such that $F\left(z_{0}\right)>0$ and

$$
F(z) \geq F\left(z_{0}\right) \exp \left(\int_{z_{0}}^{z} \frac{d \xi}{C C^{*}(\xi+h)^{\alpha}}\right)
$$

or

(b) for $z \geq 0$, the decay estimate is satisfied:

$$
-F(z) \leq-F(0) \exp \left(-\int_{0}^{z} \frac{d \xi}{C C^{*}(\xi+h)^{\alpha}}\right) .
$$

We note that the growth estimate (6.1) is of the type

$$
\exp \left(\int_{z_{0}}^{z} \frac{d \xi}{C C^{*}(\xi+h)^{\alpha}}\right)
$$

and when $\alpha \in(0,1)$ is of order $\exp z^{1-\alpha}$. Indeed, we have

$$
\exp \left(\int_{z_{0}}^{z} \frac{d \xi}{C C^{*}(\xi+h)^{\alpha}}\right)=\exp \left[\frac{1}{C C^{*}(1-\alpha)}\left((z+h)^{1-\alpha}-\left(z_{0}+h\right)^{1-\alpha}\right)\right] .
$$

Consequently, the solution as measured by $F(z)$ becomes unbounded at infinity at least to the order $\exp \left(z^{1-\alpha}\right)$.

When $\alpha=1$, we conclude that

$$
\exp \left(\int_{z_{0}}^{z} \frac{d \xi}{C C^{*}(\xi+h)}\right)=\left(\frac{(z+h)}{\left(z_{0}+h\right)}\right)^{\left(C C^{*}\right)^{-1}},
$$

so that growth is at least polynomial.

Similarly, when $0<\alpha<1$, the integral in the decay estimate (6.2) becomes

$$
\exp \left(-\int_{0}^{z} \frac{d \xi}{C C^{*}(\xi+h)^{\alpha}}\right)=\exp \left(\frac{h^{1-\alpha}}{C C^{*}(1-\alpha)}\right) \exp \left(-\left\{\frac{(z+h)^{1-\alpha}}{C C^{*}(1-\alpha)}\right\}\right)
$$

which behaves at most like $\exp \left(-z^{1-\alpha} / C C^{*}\right)$. When $\alpha=1$, we have

$$
\exp \left(-\int_{0}^{z} \frac{d \xi}{C C^{*}(\xi+h)}\right)=\left(\frac{h}{(z+h)}\right)^{\left(C C^{*}\right)^{-1}},
$$

and the decay estimate behaves as $z^{-\left(C C^{*}\right)^{-1}}$.

Remark 6.1 It is clear from expressions (6.3)-(6.6) that the optimum rate of growth or decay is achieved when the constant $C^{*}$ is chosen to have its minimum value. 
Remark 6.2 Observe that when $\gamma_{1}$ and $\gamma_{2}$ are selected to satisfy

$$
B U<A V,
$$

the rate of growth and decay becomes proportional to

$$
\begin{aligned}
\left(C^{*}\right)^{-1} & =B V^{-1} \\
& =\frac{\left(2 \lambda \mu C_{1}-a C \gamma_{1}\right)}{\left(2 \lambda \mu k+a C \gamma_{2}\right)}
\end{aligned}
$$

and the optimal rate now corresponds to the minimum value of $\gamma_{2}$ which complements the choice of $\gamma_{2}$ when condition (5.15) holds. Strict equality in (5.15) implies that the value of $\gamma_{2}$ common to (5.15) and (6.7) should be adopted. Consequently, there is no loss in confining attention to (5.15).

\subsection{Choice of constants $\gamma_{1}, \gamma_{2}$}

We recall that the constants $\gamma_{1}$ and $\gamma_{2}$ are to be chosen to ensure that

$$
A V \leq B U
$$

which in consequence leads as before to a differential inequality of type (5.16).

Suppose that $\gamma_{1}$ is chosen within the range (5.8) and that $\lambda$ and $\mu$ satisfy (5.9). We determine $\gamma_{2}$ such that (6.9) holds, which we express as the quadratic form

$$
\gamma_{2}^{2} Y_{1}+2 \gamma_{2} \frac{Y_{2}}{a C}-Y_{3} \leq 0
$$

where

$$
\begin{aligned}
Y_{1} & =\left(1-\frac{a C}{2 \gamma_{1}}\right)=A, \\
Y_{2} & =\lambda \mu\left(k-d C_{1}\right)+\frac{a C}{2}\left(d \gamma_{1}-\frac{\lambda \mu k}{\gamma_{1}}\right), \\
Y_{3} & =\left(\lambda \mu C_{1}-\gamma_{1} \frac{a C}{2}\right)=\lambda B .
\end{aligned}
$$

In terms of $Y_{1}$ and $Y_{3}$, which are positive in view of (5.8), we may write

$$
Y_{2}=\lambda \mu k Y_{1}-d Y_{3} .
$$

It follows that (6.10) is satisfied for $0<\gamma_{2} \leq \overline{\gamma_{2}}$, where $\overline{\gamma_{2}}$ is defined by

$$
\begin{aligned}
\overline{\gamma_{2}} & =\frac{\left[-Y_{2}+\sqrt{\left(Y_{2}^{2}+a^{2} C^{2} Y_{1} Y_{3}\right)}\right]}{a C Y_{1}} \\
& =\frac{\left(Y_{4}-Y_{2}\right)}{a C Y_{1}}
\end{aligned}
$$


in which

$$
\begin{aligned}
Y_{4}^{2}= & Y_{2}^{2}+a^{2} C^{2} Y_{1} Y_{3} \\
= & \gamma_{1}^{-2}\left[\gamma_{1}^{4} d^{2}\left(\frac{a C}{2}\right)^{2}+\gamma_{1}^{3} X_{1}\right. \\
& \left.+\gamma_{1}^{2} X_{2}+\gamma_{1} X_{3}+\left(\frac{a C}{2}\right)^{2}(\lambda \mu k)^{2}\right],
\end{aligned}
$$

and

$$
\begin{aligned}
& X_{1}=a C\left[\lambda \mu d\left(k-d C_{1}\right)-\frac{(a C)^{2}}{2}\right] \\
& X_{2}=\lambda^{2} \mu^{2}\left(k-d C_{1}\right)^{2}+\lambda \mu(a C)^{2}\left(C_{1}-\frac{d}{2}\right)+\frac{(a C)^{4}}{4}, \\
& X_{3}=\lambda \mu a C\left[C_{1}\left(\frac{1}{2}+\lambda \mu k d\right)-\lambda \mu k^{2}\right] .
\end{aligned}
$$

In this respect, we recall the assumptions

$$
\begin{aligned}
C_{1}= & (1-C e k)>0, \\
\frac{a C}{2}<\gamma_{1} & <\frac{2 \lambda \mu C_{1}}{a C} .
\end{aligned}
$$

The expression (6.17) along with (6.11) and (6.12) provide the explicit dependence of $\overline{\gamma_{2}}$ upon $\gamma_{1}$.

The optimal growth and decay rate corresponds to the maximum value of

$$
\begin{aligned}
\left(C^{*}\right)^{-1} & =A U^{-1} \\
& =\frac{\left(2 \gamma_{1}-a C\right)}{2 \gamma_{1} d}\left[1-\frac{a C}{\left(2 \gamma_{2} d+a C\right)}\right]
\end{aligned}
$$

which is achieved when $\gamma_{2}$ is its maximum value $\overline{\gamma_{2}}$. Substitution from (6.15) gives

$$
\begin{aligned}
\left(C^{*}\right)^{-1} & =\left(1-\frac{a C}{2 \gamma_{1}}\right) \frac{2\left(Y_{4}-Y_{2}\right)}{W} \\
& =\frac{2 Y_{1}\left(Y_{4}-Y_{2}\right)}{W} \\
& =\frac{Y_{1}}{d}-\frac{a^{2} C^{2} Y_{1}^{2}}{d W}
\end{aligned}
$$

where

$$
W=\left(a^{2} C^{2} Y_{1}+2 d\left(Y_{4}-Y_{2}\right)\right) .
$$

The value of $\gamma_{1}$ within the range (6.19) is now sought that maximises $\left(C^{*}\right)^{-1}$. Let a superposed prime denote diffferentiation with respect to $\gamma_{1}$ and differen- 
tiate (6.23) to first obtain

$$
\begin{aligned}
\left(\left(C^{*}\right)^{-1}\right)^{\prime}= & \frac{Y_{1}^{\prime}}{d}-\frac{a^{2} C^{2}}{d}\left[\frac{2 Y_{1} Y_{1}^{\prime}}{W}-Y_{1}^{2} \frac{\left(a^{2} C^{2} Y_{1}^{\prime}+2 d\left(Y_{4}^{\prime}-Y_{2}^{\prime}\right)\right)}{W^{2}}\right] \\
= & \frac{Y_{1}^{\prime}}{d}-\frac{a^{2} C^{2}}{d}\left[\frac{Y_{1} Y_{1}^{\prime}\left\{a^{2} C^{2} Y_{1}+4 d\left(Y_{4}-Y_{2}\right)\right\}-2 d Y_{1}^{2}\left(Y_{4}^{\prime}-Y_{2}^{\prime}\right)}{W^{2}}\right] \\
= & \frac{Y_{1}^{\prime}}{d}-\frac{a^{2} C^{2}}{d Y_{4} W^{2}}\left[a^{2} C^{2} Y_{1}^{2}\left(Y_{4} Y_{1}^{\prime}-d\left(Y_{1} Y_{3}\right)^{\prime}\right)+\right. \\
& \left.+2 d\left(Y_{4}-Y_{2}\right)\left(2 Y_{1} Y_{4} Y_{1}^{\prime}+Y_{1}^{2} Y_{2}^{\prime}\right)\right] .
\end{aligned}
$$

On noting the relations

$$
\begin{aligned}
Y_{1}^{\prime} & =\frac{a C}{2 \gamma_{1}^{2}}, \\
Y_{2}^{\prime} & =\lambda \mu k Y_{1}^{\prime}-d Y_{3}^{\prime} \\
& =\frac{\lambda \mu a C}{2 \gamma_{1}^{2}}+\frac{d a C}{2}, \\
Y_{3}^{\prime} & =-\frac{a C}{2}, \\
Y_{4}^{\prime} & =\frac{Y_{2} Y_{2}^{\prime}}{Y_{4}}+\frac{a^{2} C^{2}}{2 Y_{4}}\left(Y_{1} Y_{3}\right)^{\prime},
\end{aligned}
$$

we may rewrite expression $(6.25)$ as

$$
\begin{aligned}
\left(\left(C^{*}\right)^{-1}\right)^{\prime}= & \frac{a C}{2 d \gamma_{1}^{2}}-\frac{a^{3} C^{3}}{2 d} \frac{Y_{1}}{\gamma_{1}^{2} Y_{4} W^{2}}\left[Y_{1} a^{2} C^{2}\left\{\left(Y_{4}-d Y_{3}\right)+d \gamma_{1}^{2} Y_{1}\right\}+\right. \\
& \left.+2 d\left(Y_{4}-Y_{2}\right)\left\{2 Y_{4}+Y_{1}\left(\lambda \mu k+d \gamma_{1}^{2}\right)\right\}\right]
\end{aligned}
$$

We examine the sign of the derivative (6.26) at the extreme endpoints of the range $(6.19)$.

At the lower point $\gamma_{1}=a C / 2$, we have

$$
\begin{aligned}
Y_{1} & =0, \\
Y_{2} & =-d Y_{3}<0, \\
Y_{3} & =\lambda \mu C_{1}-\left(\frac{a C}{2}\right)^{2}, \\
Y_{4}^{2} & =Y_{2}^{2},
\end{aligned}
$$

and (6.27) yields

$$
\left(Y_{4}+Y_{2}\right)\left(Y_{4}-Y_{2}\right)=0,
$$

from which we conclude

$$
Y_{4}=-Y_{2}=d Y_{3},
$$


and consequently we have

$$
W=4 d Y_{3}
$$

Substitution in (6.26) gives

$$
\left(\left(C^{*}\right)^{-1}\right)^{\prime}=\frac{2}{d a C}>0 .
$$

Next consider $\gamma_{1}=2 \lambda \mu C_{1} / a C$, which leads to

$$
\begin{aligned}
Y_{1} & =1-\left(\frac{a C}{2}\right)^{2} \frac{1}{\lambda \mu C_{1}}>0, \\
Y_{2} & =\lambda \mu k Y_{1}>0, \\
Y_{3} & =0 \\
Y_{4}^{2} & =Y_{2}^{2}
\end{aligned}
$$

and again implies

$$
\left(Y_{4}+Y_{2}\right)\left(Y_{4}-Y_{2}\right)=0
$$

and consequently

$$
\begin{aligned}
Y_{4}=Y_{2} & =\lambda \mu k Y_{1}, \\
W & =a^{2} C^{2} Y_{1} .
\end{aligned}
$$

Insertion into (6.26) leads to

$$
\begin{aligned}
\left(\left(C^{*}\right)^{-1}\right)^{\prime} & =\frac{a C}{2 d \gamma_{1}^{2}}\left[1-\frac{a^{2} C^{2} Y_{1}}{\lambda \mu k Y_{1}}\left(\frac{Y_{1} a^{2} C^{2}\left\{\lambda \mu k Y_{1}+d Y_{1}\left(\frac{2 \lambda \mu C_{1}}{a C}\right)^{2}\right\}}{a^{4} C^{4} Y_{1}^{2}}\right)\right] \\
& =\frac{a C}{2 d \gamma_{1}^{2}}\left[1-\frac{1}{Y_{1}^{2}}\left(Y_{1}\left\{Y_{1}+\frac{4 d \lambda \mu C_{1}^{2}}{a^{2} C^{2} k}\right\}\right)\right] \\
& =-\frac{\lambda \mu C_{1}^{2}}{a C k Y_{1}} \\
& <0 .
\end{aligned}
$$

In consequence, the derivative of $\left(C^{*}\right)^{-1}$ must vanish at least once in the interval (6.19), the first zero being a (local) maximum for $\left(C^{*}\right)^{-1}$. Other maxima also may occur in the same interval. Let $\overline{\gamma_{1}}$ denote the greatest such maximum. Then the optimum growth and decay rate is obtained for $\overline{\gamma_{1}}$ which may be calculated, for example, by standard computer programs. Details are omitted as they are beyond the intended scope of the paper. Even so, optimum growth and decay rates remain elusive since the maximum value of the constant $\gamma_{1}$ can be chosen arbitrarily close, but not exactly equal, to the upper bound in the range (5.8)

Less general methods that nevertheless lead to explicit rates are described in the Appendix, where the mean value of $\gamma_{1}$ in the range (5.8) is adopted as a compromise in (A.9). 


\section{Implications of the decay estimate}

\subsection{Energy decay}

Under conditions sufficient for decay, we infer from (6.2) that $F(z) \leq 0, z \geq 0$ and that

$$
\lim _{z \rightarrow \infty} F(z)=0 .
$$

Integration of inequality (5.5) yields

$$
\begin{aligned}
-F(z) \geq & A \int_{z}^{\infty}\left[\int_{D(\eta)} d_{i j k h} u_{i, j} u_{k, h} d a\right. \\
& \left.+A^{-1} B \Lambda(\eta) \int_{D(\eta)} k_{i j} \theta_{, i} \theta_{, j} d a\right] d \eta \\
\geq & A \int_{\Omega(z)}\left[d_{i j k h} u_{i, j} u_{k, h}\right. \\
& \left.+A^{-1} B \Lambda(0) k_{i j} \theta_{, i} \theta_{, j}\right] d a d \eta,
\end{aligned}
$$

since $\Lambda(z)=\lambda(z+h)^{\alpha} \geq \lambda h^{\alpha}=\Lambda(0)$, and where $\Omega(z)$ is defined in (2.1).

Define the total energy to be

$$
E(z)=\int_{\Omega(z)}\left(d_{i j k h} u_{i, j} u_{k, h}+A^{-1} B \Lambda(0) k_{i j} \theta_{, i} \theta_{, j}\right) d a d \eta .
$$

Then from (7.3) and (6.2), we conclude that

$$
\begin{aligned}
E(z) & \leq-A^{-1} F(z) \\
& \leq-A^{-1} F(0) \exp \left(-\int_{0}^{z} \frac{d \xi}{C C^{*}(\xi+h)^{\alpha}}\right)
\end{aligned}
$$

In particular, we deduce from (7.5) the further estimate

$$
E(0) \leq-A^{-1} F(0)
$$

\subsection{Asymptotic Limits. First method}

The decay estimates (6.2) and (7.5) only implicitly describe the asymptotic behaviour of the practically important positive-definite terms

$$
\begin{aligned}
J_{1}(z) & =\int_{D(z)} d_{i j k l} u_{i, j} u_{k, l} d a, \\
J_{2}(z) & =\int_{D(z)} k_{i j} \theta_{, i} \theta_{, j} d a, \\
J_{3}(z) & =\Lambda(z) \int_{D(z)} k_{i j} \theta_{, i} \theta_{, j} d a .
\end{aligned}
$$


Such information is easily extracted from inequalities (7.2) and (7.5) for solutions in $W^{1,2}(\Omega)$. Indeed, (7.5) implies that

$$
\lim _{z \rightarrow \infty} J_{\gamma}(z)=0, \quad \gamma=1,2,
$$

while (7.2) implies that

$$
\lim _{z \rightarrow \infty} J_{3}(z)=0
$$

and from (2.8) that

$$
\lim _{z \rightarrow \infty} \int_{D(z)} \theta^{2} d a=\lim _{z \rightarrow \infty} \Lambda(z) J_{2}(z)=\lim _{z \rightarrow \infty} J_{3}(z)=0 .
$$

We use the asymptotic behaviour specified in (7.8) to derive a decay estimate for the mean-square cross-sectional measure of the temperature $\theta$. Let

$$
H(z)=\int_{\Omega(z)} \theta^{2} d a d \eta
$$

so that by (7.8) we have

$$
\begin{aligned}
-H^{\prime}(z) & =\int_{D(z)} \theta^{2} d a \\
& =-2 \int_{\Omega(z)} \theta \theta_{, 1} d a d \eta \\
& \leq 2\left(\int_{\Omega(z)} \theta^{2} d a d \eta\right)^{1 / 2}\left(\int_{\Omega(z)} \theta_{, 1}^{2} d a d \eta\right)^{1 / 2}
\end{aligned}
$$

which on rearrangement and appeal to (7.5) gives the differential inequality

$$
\begin{aligned}
-\frac{H^{\prime}(z)}{H^{1 / 2}(z)} & \leq \frac{2}{k_{1}^{1 / 2}}\left(\int_{\Omega(z)} k_{i j} \theta_{, i} \theta_{, j} d a d \eta\right)^{1 / 2} \\
& \leq\left[\frac{-4 F(0)}{k_{1} B \Lambda(0)}\right]^{1 / 2} \exp \left(-\frac{1}{2} \int_{0}^{z} \frac{d \xi}{C C^{*}(\xi+h)^{\alpha}}\right) \\
& =\left[\frac{-4 F(0)}{k_{1} B \Lambda(0)}\right]^{1 / 2} \exp \left(\frac{h^{1-\alpha}}{2 C C^{*}(1-\alpha)}\right) \exp \left(-\frac{(z+h)^{1-\alpha}}{2 C C^{*}(1-\alpha)}\right) .
\end{aligned}
$$

where $0<\alpha<1$. When $\alpha=1$, the previous inequality reduces to

$$
-\frac{H^{\prime}(z)}{H^{1 / 2}(z)} \leq\left[\frac{-4 F(0)}{k_{1} B \Lambda(0)}\right]^{1 / 2}\left(\frac{h}{(z+h)}\right)^{\left(2 C C^{*}\right)^{-1}} .
$$

On using $[3, \S 3.381,3$, p.364] to evaluate the integral, we may integrate over $(z, \infty)$ to obtain for $0<\alpha<1$ the estimate

$$
\begin{aligned}
H^{1 / 2}(z) \leq & 4\left[\frac{-F(0)}{k_{1} B \Lambda(0)}\right]^{1 / 2} \exp \left(\frac{h^{1-\alpha}}{2 C C^{*}(1-\alpha)}\right)\left[\frac{1}{(z+h)(1-\alpha)}\right] \times \\
& \times \Gamma\left(\frac{1}{(1-\alpha)}, \frac{(z+h)^{1-\alpha}}{2 C C^{*}(1-\alpha)}\right) .
\end{aligned}
$$


When $\alpha=1$ and $2 C C^{*}<1$, the estimate becomes

$$
H^{1 / 2}(z) \leq 4\left[\frac{-F(0)}{k_{1} B \Lambda(0)}\right]^{1 / 2} \frac{h^{\left(\frac{1}{2 C C_{i}}\right)}}{\left(1-2 C^{-1}\left(C^{*}\right)^{-1}\right)}\left[(z+h)^{\left(1-\left[2 C C^{*}\right]^{-1}\right)}\right],
$$

where the incomplete gamma function $\Gamma(\gamma, x)$ is defined by

$$
\Gamma(\gamma, x)=\int_{0}^{x} e^{-\eta} \eta^{\gamma-1} d \eta .
$$

The corresponding integral in (7.12) is divergent for $2 C C^{*} \geq 1$.

An estimate for the mean-square cross-sectional measure of the temperature is obtained by combining (7.10) with (7.5) and either (7.11) or (7.12).

Although a similar argument may be applied to the displacement, a slightly different treatment is presented in the next section.

\subsection{Asymptotic Limits. Second method}

A second derivation of asymptotic behaviour is based upon an application of L'Hopital's Theorem to (7.5) and leads to the limit

$$
\begin{aligned}
& \lim _{z \rightarrow \infty} C C^{*}(z+h)^{\alpha} \exp \frac{(z+h)^{1-\alpha}}{C C^{*}(1-\alpha)}\left[\int_{D(z)}\left\{d_{i j k l} u_{i, j} u_{k, l}+A^{-1} B \Lambda(0) k_{i j} \theta_{, i} \theta_{, j}\right\} d a\right] \\
\leq & -A^{-1} F(0) .
\end{aligned}
$$

In consequence, on appealing to the positive-definite conditions (3.8) and (3.11), we conclude that

$$
\begin{aligned}
\lim _{z \rightarrow \infty}(z+h)^{\alpha} \exp \frac{(z+h)^{1-\alpha}}{C C^{*}(1-\alpha)} \int_{D(z)} u_{i, j} u_{i, j} d a & =-A^{-1} F(0), \\
\lim _{z \rightarrow \infty}(z+h)^{\alpha} \exp \frac{(z+h)^{1-\alpha}}{C C^{*}(1-\alpha)} \int_{D(z)} \theta_{, i} \theta_{, i} d a & =-A^{-1} F(0),
\end{aligned}
$$

so that the displacement and temperature tend asymptotically to respective constants which vanish by virtue of the boundary conditions (3.13).

Inequality (2.8) may be used to derive from (7.15) the improved asymptotic decay behaviour

$$
\lim _{z \rightarrow \infty}(z+h)^{-\alpha} \exp (z+h)^{1-\alpha} \int_{D(z)} u_{i} u_{i} d a \leq M_{1},
$$

where $M_{1}$ is a non-negative constant.

To derive an estimate for the mean-square cross-sectional integral of the displacement, we introduce the function

$$
P(z)=\int_{\Omega(z)} u_{i} u_{i} d a d \eta
$$


which by (7.17) is convergent and indeed

$$
\lim _{z \rightarrow \infty} P(z)=0 .
$$

Differentiation followed by appeal to (7.19) and Schwarz's inequality yields

$$
\begin{aligned}
-P^{\prime}(z) & =\int_{D(z)} u_{i} u_{i} d a \\
& =-2 \int_{\Omega(z)} u_{i} u_{i, 1} d a d \eta \\
& \leq 2\left(\int_{\Omega(z)} u_{i} u_{i} d a d \eta\right)^{1 / 2}\left(\int_{\Omega(z)} u_{i, 1} u_{i, 1} d a d \eta\right)^{1 / 2} \\
& \leq 2 P^{1 / 2}(z)\left(\int_{\Omega(z)} u_{i, j} u_{i, j} d a d \eta\right)^{1 / 2} \\
& \leq 2 d_{1}^{-1 / 2} P^{1 / 2}(z)\left(\int_{\Omega(z)} d_{i j k l} u_{i, j} u_{k, l} d a d \eta\right)^{1 / 2} \\
& \leq 2 d_{1}^{-1 / 2} P^{1 / 2}(z) E^{1 / 2}(z) .
\end{aligned}
$$

Replacement of the energy by the decaying upper bound (7.5) yields the differential inequality

$$
-\frac{P^{\prime}(z)}{P^{1 / 2}(z)} \leq 2\left[\frac{-F(0)}{d_{1} A}\right]^{1 / 2} \exp \left(-\frac{1}{2} \int_{0}^{z} \frac{d \xi}{C C^{*}(\xi+h)^{\alpha}}\right),
$$

where $0<\alpha<1$, with a corresponding upper bound for $\alpha=1$.

Integration of these inequalities and their subsequent treatment is accomplished in the manner just described for the temperature, and leads to estimates for $P(z)$ and for the mean-square cross-sectional measure $P^{\prime}(z)$.

\section{Implications of the growth estimate}

We next discuss implications of the growth estimate (6.1) for functions related to the total energy. Define the function $\Pi_{1}(z)$ to be

$$
\Pi_{1}(z)=\int_{0}^{z} \int_{D(\eta)}\left(d_{i j k h} u_{i, j} u_{k, h}+\beta_{i j} u_{i, j} \theta\right) d \eta d a+\mu \int_{0}^{z} G(\eta) d \eta .
$$

It immediately follows from (5.2) that

$$
\begin{aligned}
\Pi_{1}(z) & =F(z)-F(0) \\
& \geq-F(0)+F\left(z_{0}\right) \exp \left(\int_{z_{0}}^{z} \frac{d \xi}{C C_{i}(\xi+h)^{\alpha}}\right),
\end{aligned}
$$


where $z \geq z_{0} \geq 0$ and $z_{0}$ is such that $F\left(z_{0}\right)>0$.

The function $G(z)$ defined in (4.1) has at least polynomial growth. Nevertheless, we conclude from estimate (8.2) that the function $\Pi_{1}(z)$ becomes exponentially unbounded as $z$ tends to infinity which, with respect to the integrals appearing in (8.1), implies that either one or both possess the same exponentially growing lower bound. The conclusion may be refined when $0<\alpha<1$ by considering the function

$$
\Pi_{2}(z)=A_{1} \int_{0}^{z} \int_{D(\eta)}\left(d_{i j k h} u_{i, j} u_{k, h}+\Lambda_{0}(\eta) k_{i j} \theta_{, i} \theta_{, j}\right) d \eta d a,
$$

where

$$
A_{1}=\left[1+\frac{C}{2}\left(e k+\sqrt{\left(e^{2} k^{2}+a^{2}\right)}\right)\right] .
$$

On using the upper instead of the lower bounds from (4.4) - (4.8), we may conclude from (5.2) that

$$
\Pi_{2}(z) \geq F(z)-F(0), \quad z \geq z_{0} \geq 0 .
$$

Consequently, either integral or both in (8.3) possess exponentially increasing lower bounds

The results of this section and of Section 7.1 may be summarised in the following:

Corollary 1. Assume that $\left(u_{i}, \theta\right)$ is a solution to the boundary value problem under consideration and let $0<\alpha<1$. Then the following alternatives hold:

Either

(a) The function $\Pi_{2}(z)$ defined at (8.3) becomes unbounded, or

(b) The energy function $E(z)$ satisfies the decay estimate (7.5).

\section{The amplitude}

It is evident that the rate of decay depends solely upon data. In contrast, the decay estimate (7.5) obtained in the previous section is impractical because dependence of the amplitude $-F(0)$ on the data is not explicit. The aim of this section is to obtain an upper bound for $-F(0)$ in terms of the data (3.13) and (3.14), but for a general family of regions $\Omega$ that includes those previously considered.

Indeed, the regions $\Omega$ are such that the cross-sections $D(z)$ satisfy

$$
D(z)=\zeta(z) D(0), \quad z \geq 0,
$$

where the $C^{1}$-function $\zeta:[0, \infty) \rightarrow(0, \infty)$ satisfies $\zeta(0)=1$, and $\zeta(z),\left|\zeta^{\prime}(z)\right|$ are bounded by a polynomial $Q(z)$ for every $z \geq 0$. That is, points in $D(z)$ are given by $\left(z, \zeta(z) x_{2}, \zeta(z) x_{3}\right)$ where $\left(0, x_{2}, x_{3}\right) \in D(0)$. Moreover, we assume that the correspondence is bijective. 
The examples discussed in Section 2 involve regions satisfying (9.1) with the function $\zeta(z)$ given by

$$
\zeta(t)=\frac{(t+h)^{\alpha}}{h^{\alpha}}, \quad \alpha \in(0,1] .
$$

Denote by $\left(v_{i}, \varphi\right)$ smooth functions satisfying the boundary conditions (3.13), (3.14) whose asymptotic decay is sufficiently fast ${ }^{1}$. Substitution in (5.1) gives

$$
-F(0)=\int_{D(0)}\left(d_{i j k l} u_{k, l}+\beta \theta\right) v_{i} n_{j} d a+\mu \Lambda(0) \int_{D(0)} k_{i j} \theta_{, i} \varphi n_{j} d a,
$$

which after an integration by parts yields

$$
\begin{aligned}
-F(0)= & \int_{\Omega}\left(d_{i j k h} u_{i, j} v_{k, h}+\beta_{i j} v_{i, j} \theta\right) d a d \eta+\mu \Lambda(0) \int_{\Omega} k_{i j} \theta_{, i} \varphi_{, j} d a d \eta \\
& +\tilde{F}(\infty),
\end{aligned}
$$

where by hypothesis $F(0)<0$. Furthermore, we have set

$$
\tilde{F}(z)=\int_{D(z)}\left(d_{i j k l} u_{k, l}+\beta_{i j} \theta\right) v_{i} n_{j} d a+\mu \Lambda(0) \int_{D(z)} k_{i j} \theta_{, i} \varphi d a .
$$

Schwarz's inequality gives

$$
\begin{aligned}
\tilde{F}(z) \leq & \left(\int_{D(z)} d_{i j k l} u_{i, j} u_{k, l} d a \int_{D(z)} d_{i j k l} v_{i} v_{k} n_{j} n_{l} d a\right)^{1 / 2} \\
& +\left(\int_{D(z)} \beta_{i j} \beta_{i j} \theta^{2} d a \int_{D(z)} v_{i} v_{i} n_{j} n_{j} d a\right)^{1 / 2} \\
& +\mu \Lambda(0)\left(\int_{D(z)} k_{i j} \theta_{, i} \theta_{, j} d a \int_{D(z)} k_{i j} \varphi^{2} n_{i} n_{i} d a\right)^{1 / 2} \\
\leq & {\left[\left(\tilde{d} \int_{D(z)} d_{i j k l} u_{i, j} u_{k, l} d a\right)^{1 / 2}+\left(\tilde{\beta} \int_{D(z)} \theta^{2} d a\right)^{1 / 2}\right]\left(\int_{D(z)} v_{i} v_{i} d a\right)^{1 / 2} } \\
& +\mu \Lambda(0)\left(\int_{D(z)} k_{i j} \theta_{, i} \theta_{, j} d a \int_{D(z)} \tilde{k} \varphi^{2} n_{i} n_{i} d a\right)^{1 / 2} \cdot
\end{aligned}
$$

We now suppose that

$$
\begin{gathered}
\lim _{z \rightarrow \infty} \int_{D(z)} v_{i} v_{i} d a \leq M_{2}^{2}, \\
\lim _{z \rightarrow \infty} \int_{D(z)} \varphi^{2} d a \leq M_{3}^{2},
\end{gathered}
$$

\footnotetext{
${ }^{1}$ This assumption can be made explicit. For instance we can assume that $\left(v_{i}, \varphi\right)$ tend to zero exponentially in the $x_{1}$ component, and uniformly with respect to $\left(x_{2}, x_{3}\right)$
} 
where $M_{2}, M_{3}$ are specified constants. Consequently, by virtue of the asymptotic behaviour (7.7), we conclude

$$
\tilde{F}(\infty)=\lim _{z \rightarrow \infty} \tilde{F}(z)=0
$$

and therefore (9.3) becomes

$$
-F(0)=\int_{\Omega}\left(d_{i j k h} u_{i, j} v_{k, h}+\beta_{i j} v_{i, j} \theta\right) d a d \eta+\mu \Lambda(0) \int_{\Omega} k_{i j} \theta_{, i} \varphi_{, j} d a d \eta,
$$

which on noting (7.6) and successively applying the Schwarz and arithmeticgeometric mean inequalities, we conclude that

$$
\begin{aligned}
-F(0) \leq & \left(\int_{\Omega} d_{i j k h} u_{i, j} u_{k, h} d a d \eta \int_{\Omega} d_{i j k h} v_{i, j} v_{k, h} d a d \eta\right)^{1 / 2} \\
& +\left(\int_{\Omega}(z+h)^{2 \alpha} v_{i, j} v_{i, j} d a d \eta \int_{\Omega}(z+h)^{-2 \alpha} \beta_{i j} \beta_{i j} \theta^{2} d a d \eta\right)^{1 / 2} \\
& +\mu \Lambda(0)\left(\int_{\Omega} k_{i j} \theta_{, i} \theta_{, j} d a d \eta\right)^{1 / 2}\left(\int_{\Omega} k_{i j} \varphi_{, i} \varphi_{, j} d a d \eta\right)^{1 / 2} \\
\leq & \frac{\epsilon_{1}}{2} \int_{\Omega} d_{i j k h} u_{i, j} u_{k, h} d a d \eta+\frac{1}{2 \epsilon_{1}} \int_{\Omega} d_{i j k h} v_{i, j} v_{k, h} d a d \eta \\
& +\frac{1}{2 \lambda \epsilon_{2}} \int_{\Omega} \Lambda(\eta) v_{i, j} v_{i, j} d a d \eta+\frac{C^{2} \tilde{\beta}_{2}}{2 k_{1}} \int_{\Omega} k_{i j} \theta_{, i} \theta_{, j} d a d \eta \\
& +\frac{\mu \Lambda(0) \epsilon_{3}}{2} \int_{\Omega} k_{i j} \theta_{, i} \theta_{, j} d a d \eta+\frac{\mu \Lambda(0)}{2 \epsilon_{3}} \int_{\Omega} k_{i j} \varphi_{, i} \varphi_{, j} d a d \eta \\
= & \frac{\epsilon_{1}}{2} \int_{\Omega} d_{i j k l} u_{i, j} u_{k, l} d a d \eta+\left[\frac{C^{2} \widetilde{\beta} \epsilon_{2}}{2 k_{1}}+\frac{\mu \Lambda(0) \epsilon_{3}}{2}\right] \int_{\Omega} k_{i j} \theta_{, i} \theta_{, j} d a d \eta \\
& +\frac{1}{2 \epsilon_{1}} \int_{\Omega} d_{i j k l} v_{i, j} v_{k, l} d a \eta+\frac{1}{2 \lambda \epsilon_{2}} \int_{\Omega} \Lambda(z) v_{i, j} v_{i, j} d a d \eta+\frac{\mu \Lambda(0)}{2 \epsilon_{3}} \int_{\Omega} k_{i j} \varphi_{, i} \varphi_{, j} d a d \eta,
\end{aligned}
$$

where the arbitrary positive constants $\epsilon_{i}, i=1, \ldots, 3$ are chosen to be

$$
\begin{aligned}
\epsilon_{1} & =A, \\
\epsilon_{2} & =\frac{k_{1} B \Lambda(0)}{2 C^{2} \widetilde{\beta}}, \\
\epsilon_{3} & =\frac{B}{2 \mu} .
\end{aligned}
$$

Substitution yields

$$
\begin{aligned}
\frac{\epsilon_{1}}{2} \int_{\Omega} d_{i j k l} u_{i, j} u_{k, l} d a d \eta & +\left[\frac{C^{2} \widetilde{\beta} \epsilon_{2}}{2 k_{1}}+\frac{\mu \Lambda(0) \epsilon_{3}}{2}\right] \int_{\Omega} k_{i j} \theta_{, i} \theta_{, j} d a d \eta \\
& =\frac{A}{2} \int_{\Omega} d_{i j k l} u_{i, j} u_{k, l} d a d \eta+\frac{B \Lambda(0)}{2} \int_{\Omega} k_{i j} \theta_{, i} \theta_{, j} d a d \eta \\
& =A E(0) / 2 \\
& \leq-F(0) / 2
\end{aligned}
$$


where (7.4) is used and the last inequality follows from (7.6).

An upper bound for $-F(0)$ in terms of data can then be established in the form

$$
\begin{aligned}
-F(0) \leq & \frac{1}{\epsilon_{1}} \int_{\Omega} d_{i j k h} v_{i, j} v_{k, h} d a d \eta+\frac{1}{\lambda \epsilon_{2}} \int_{\Omega} \Lambda(\eta) v_{i, j} v_{i, j} d a d \eta \\
& +\frac{\mu \Lambda(0)}{\epsilon_{3}} \int_{0}^{\infty} \int_{\Omega} k_{i j} \varphi_{, i} \varphi_{, j} d a d \eta .
\end{aligned}
$$

An explicit estimate is derived on taking

$$
\begin{gathered}
v_{i}\left(x_{1}, x_{2}, x_{3}\right)=f_{i}\left(x_{2} \zeta^{-1}\left(x_{1}\right), x_{3} \zeta^{-1}\left(x_{1}\right)\right) \exp \left(-\omega x_{1}\right), \\
\varphi\left(x_{1}, x_{2}, x_{3}\right)=g\left(x_{2} \zeta^{-1}\left(x_{1}\right), x_{3} \zeta^{-1}\left(x_{1}\right)\right) \exp \left(-\omega x_{1}\right),
\end{gathered}
$$

where $\omega$ is a positive constant, $\zeta(z)$ is the function appearing in (9.1), and the prescribed functions $f_{i}(.,),. g($.$) are those appearing in (3.14).$

In particular, we have for $\delta=2,3$ :

$$
\begin{gathered}
v_{i, 1}=a_{i} \exp \left(-\omega x_{1}\right), \\
a_{i}=-\omega f_{i}\left(x_{2} \zeta^{1}\left(x_{1}\right), x_{3} \zeta^{-1}\left(x_{1}\right)\right)-\frac{x_{\delta} \zeta^{\prime}\left(x_{1}\right)}{\zeta^{2}\left(x_{1}\right)} f_{i, \delta}\left(x_{2} \zeta^{-1}\left(x_{1}\right), x_{3} \zeta^{-1}\left(x_{1}\right)\right), \\
v_{i, \delta}=\frac{1}{\zeta\left(x_{1}\right)} f_{i, \delta}\left(x_{2} \zeta^{-1}\left(x_{1}\right), x_{3} \zeta^{-1}\left(x_{1}\right)\right) \exp \left(-\omega x_{1}\right), \\
\varphi_{, 1}=m \exp \left(-\omega x_{1}\right), \\
m=-\omega g\left(x_{2}^{1} \zeta^{-1}\left(x_{1}\right), x_{3} \zeta^{-1}\left(x_{1}\right)\right)-\frac{x_{\delta} \zeta^{\prime}\left(x_{1}\right)}{\zeta^{2}\left(x_{1}\right)} g_{, \delta}\left(x_{2} \zeta^{-1}\left(x_{1}\right), x_{3} \zeta^{-1}\left(x_{1}\right)\right),
\end{gathered}
$$

and

$$
\varphi_{, \delta}=\frac{1}{\zeta\left(x_{1}\right)} g_{, \delta}\left(x_{2} \zeta^{-1}\left(x_{1}\right), x_{3} \zeta^{-1}\left(x_{1}\right)\right) \exp \left(-\omega x_{1}\right) .
$$

Appeal to (3.9)-(3.12), and (3.19) combined with an easy substitution then leads to

$$
\begin{aligned}
\int_{\Omega} d_{i j k l} v_{i, j} v_{k, l} d a d \eta & \leq \widetilde{d} \int_{\Omega} v_{i, j} v_{i, j} d a d \eta \\
& =\widetilde{d} \int_{\Omega} \exp (-2 \omega \eta)\left[a_{i} a_{i}+\zeta^{-2}(\eta) f_{i, \delta} f_{i, \delta}\right] d a d \eta \\
\int_{\Omega} \Lambda(\eta) v_{i, j} v_{i, j} d a d \eta= & \int_{\Omega} \Lambda(\eta) \exp (-2 \omega \eta)\left[a_{i} a_{i}+\zeta^{-2}(\eta) f_{i, \delta} f_{i, \delta}\right] d a d \eta \\
\int_{\Omega} k_{i j} \varphi_{, i} \varphi_{, j} d a d \eta= & \int_{\Omega} \exp (-2 \omega \eta)\left[k_{11} m^{2}+2 k_{1 \delta} m \zeta^{-1}(\eta) g_{, \delta}\right. \\
& \left.+k_{\gamma \delta} \zeta^{-2} g_{, \gamma} g_{, \delta}\right] d a d \eta .
\end{aligned}
$$

The indefinite integrals appearing in these estimates converge whenever $\omega$ is a positive constant, while the desired result is obtained by substituting these estimates in (9.9). This upper bound can be optimised by selecting the value of $\omega$ to minimise the bound. 


\section{Comparison of estimated growth and decay rates in the uncoupled problem}

It is of interest to compare the estimated growth and decay rates for the uncoupled mechanical and thermal problems. Precise dependence upon the thermal coupling tensor is postponed to a later study. The argument developed in Section 5 is here applied simultaneously to both uncoupled problems, before each problem is separately treated. The estimated rates obtained are then compared and conditions deduced for the optimum rate. The weight function $\Lambda(z)$ moderates the rates in all cases, but does not affect the comparative relations between them. For simplicity, only the case $0<\alpha<1$ is considered.

The mechanical and thermal problems become uncoupled when $\beta_{i j}=0$ so that (3.10) gives $\widetilde{\beta}=0$, while the respective definitions (3.17), (5.6), and (5.7) give $a=b=0$, and $A=1, B=\mu C_{1}$. The constant $\gamma_{1}$ is therefore redundant. Further inspection shows that $I_{2}(z)=0, U=d$, and $V=\mu k$. Consequently, the constant $\gamma_{2}$ is likewise redundant.

To simultaneously apply the method of Section 5 , suppose first that $d C_{1} \geq k$, or equivalently that

$$
k \leq \frac{d}{(1+\operatorname{de} C)}<\frac{1}{e C},
$$

which implies that, but is not implied by, $k<d$. The second inequality is satisfied for sufficiently small $e C$.

The differential inequality (5.16) reduces to

$$
|F(z)| \leq d C(z+h)^{\alpha} F^{\prime}(z),
$$

in which

$$
F(z)=\int_{D(z)} d_{i 1 k l} u_{k, l} u_{i} d a+\mu \Lambda(z) \int_{D(z)} k_{i 1} \theta_{, i} \theta d a .
$$

Integration and subsequent discussion of the differential inequality (10.2) repeats the earlier procedures allowing details to be safely omitted. Of present importance is the corresponding estimated growth and decay rate $R_{1}$ which, easilty obtained from (5.16) and the discussion of Section 6 , is given by

$$
R_{1}=\frac{1}{d C(1-\alpha)} .
$$

Next, suppose that $d C_{1} \leq k$, which after rearrangement may be expressed as

$$
k \geq \frac{d}{(1+d e C)},
$$

and is satisfied for sufficiently large $e C$ irrespective of the magnitudes of $k$ and $d$. The differential inequality (5.16) becomes

$$
|F(z)| \leq k C C_{1}^{-1}(z+h)^{\alpha} F^{\prime}(z) .
$$


The estimated growth and decay rate $R_{2}$ now becomes proportional to $B V^{-1}$ and by Section 6 is given explicity by

$$
R_{2}=\frac{C_{1}}{k C(1-\alpha)},
$$

where in accordance with (3.19), $k$ must be restricted to the range

$$
\frac{d}{(1+d e C)} \leq k<\frac{1}{e C}
$$

We conclude that the rates $R_{1}$ and $R_{2}$ are in the proportion

$$
R_{1}=\frac{k}{d C} R_{2}
$$

but it is worth remarking that the rates are determined under different conditions (10.1) and (10.5).

Complete uncoupling is achieved by separate consideration of the mechanical and thermal problems.

The mechanical problem corresponds to $\beta_{i j}=0, k_{i j}=0$, and so (3.10) and (3.16) imply $\widetilde{\beta}=k=0$, while (2.4) implies $C_{1}=1$, and (4.1) gives $G(z)=0$. Moreover, the notation of Section 5 simplifies to $F(z)=I_{1}(z), I_{2}(z)=I_{3}(z)=$ 0 , and the differential inequality (5.16) becomes

$$
|F(z)| \leq d C(z+h)^{\alpha} F^{\prime}(z),
$$

leading to an estimated growth and decay rate $R_{M}$ given by

$$
R_{M}=\frac{1}{d C(1-\alpha)} .
$$

Note that $R_{M}$ is identical to $R_{1}$, but does not require (10.1) which is trivially satisfied.

For the uncoupled thermal problem, we have $\beta_{i j}=d_{i j k l}=0$, and $\widetilde{\beta}=d=0$ follows from (3.10) and (3.16). Now, however, $C_{1}$ continues to be defined by (2.4), which reduces to

$$
0<k<(e C)^{-1}
$$

while $G(z)$ remains defined by (4.1). The first inequality in (10.8) is satisfied by our assumptions on the heat conduction tensor. Furthermore, $F(z)=$ $I_{3}(z), I_{1}(z)=I_{2}(z)=0$, and the differential inequality becomes

$$
|F(z)| \leq k C C_{1}^{-1}(z+h)^{\alpha} F^{\prime}(z),
$$

which leads to the growth and decay rate

$$
R_{T}=\frac{C_{1}}{k C(1-\alpha)} .
$$

Consequently, $R_{T}=R_{2}$. 
The rates $R_{M}$ and $R_{T}$ are related by

$$
R_{M}=\frac{k}{d C_{1}} R_{T},
$$

and therefore are in the same ratio (10.6) as $R_{1}$ and $R_{2}$. After substitution from (3.18), we conclude that $R_{M}$ is greater than, equal to, or less than $R_{T}$ according as $k$ is greater than, equal to, or less than

$$
\frac{d}{(1+d e C)} \text {. }
$$

These conclusions establish that

$$
\begin{aligned}
& k<\frac{d}{(1+d e C)} \quad \Rightarrow \quad R_{1}=R_{M}<R_{T}, \\
& k>\frac{d}{(1+d e C)} \quad \Rightarrow \quad R_{M}>R_{T}=R_{2}, \\
& k=\frac{d}{(1+d e C)} \quad \Rightarrow \quad R_{M}=R_{T}=R_{1}=R_{2} .
\end{aligned}
$$

In particular, observe that $k<d$ implies the second and third of these relations, but not the first. On the other hand, $k \geq d$ implies the second relation, but neither the first nor third. Although similar comparison with estimated rates in the coupled problem discussed in Section 6.1 is comparatively straightforward, the expressions are complicated and consequently further analysis is not undertaken.

\section{References}

[1] D'Apice, C., Ciarletta, M. and Chiriţă, S. (2011). Saint-Venant decay rates for an inhomogeneous isotropic linear thermoelastic strip. J.Math.Anal.Appl., 381,121-133.

[2] Flavin,J. N., Knops, R. J. and Payne, L. E. (1989). Decay estimates for the constrained elastic cylinder of variable cross-section. Q.Appl. Math., 47, 325-350.

[3] Gradshteyn, I. S. and Ryzhik, I. M. (1995). Table of Integrals, Series, and Products (ed. A. Jeffrey). Fifth Edition. (Translated from the Russian). Boston San Diego New York: Academic Press.

[4] Hardy, G.H., Littlewood, J. E. and Pólya, G. (1952). Inequalities. Second Edition. Cambridge: Cambridge University Press.

[5] Knops, R. J., Rionero, S., and Payne, L. E. (1990). Saint-Venant's principle on unbounded domains. Proc. Roy. Soc. Edin., 115A,319-336.

[6] Lupoli, C. (1993). A Phragmén-Lindelöf principle for the thermoelastic cylinder of variable cross-section. Meccanica 28, 315-325. 


\section{A Appendix: Particular choices of $\gamma_{1}, \gamma_{2}$}

This Appendix presents three alternative methods for selecting the positive constants $\gamma_{1}, \gamma_{2}$, and lead to different growth and decay rates. Of course, $\gamma_{1}$ is assumed always to satisfy condition (5.8), but additional conditions are also imposed which curtail the applicability of the general procedure.

\section{A.1 Method 1}

The first selection of $\gamma_{1}$ and $\gamma_{2}$ is taken to ensure that

$$
\begin{aligned}
& A=B, \\
& U=V,
\end{aligned}
$$

and yields the choice

$$
\begin{aligned}
\gamma_{1} & =-\frac{\lambda\left(1-\mu C_{1}\right)}{a C}+\lambda \sqrt{\left[\left(\frac{\left(1-\mu C_{1}\right)}{a C}\right)^{2}+\frac{1}{\lambda}\right]}, \\
A & =B=\frac{1}{2}\left(1+\mu C_{1}\right)-\frac{a C}{2} \sqrt{\left[\left(\frac{\left(1-\mu C_{1}\right)}{a C}\right)^{2}+\frac{1}{\lambda}\right]}, \\
\gamma_{2} & =-\frac{\lambda(\mu k-d)}{a C}+\lambda \sqrt{\left[\left(\frac{(\mu k-d)}{a C}\right)^{2}+\frac{1}{\lambda}\right]} \\
U & =V=\frac{1}{2}(\mu k+d)+\frac{a C}{2} \sqrt{\left[\left(\frac{(\mu k-d)}{a C}\right)^{2}+\frac{1}{\lambda}\right]}
\end{aligned}
$$

The corresponding growth and decay rates become proportional to

$$
\begin{aligned}
\left(C^{*}\right)^{-1}= & A U^{-1} \\
= & \frac{\lambda}{\left(4 \lambda \mu k d-a^{2} C^{2}\right)}\left[\left(1+\mu C_{1}\right)-a C \sqrt{\left\{\left(\frac{\left(1-\mu C_{1}\right)}{a C}\right)^{2}+\frac{1}{\lambda}\right\}}\right] \\
& \times\left[(\mu k+d)-a C \sqrt{\left.\left\{\left(\frac{(\mu k-d)}{a C}\right)^{2}+\frac{1}{\lambda}\right\}\right]} \frac{1}{\left.\lambda\left(1+\mu C_{1}\right)+a C \sqrt{\left\{\left(\frac{\left(1-\mu C_{1}\right)}{a C}\right)^{2}+\frac{1}{\lambda}\right\}}\right]}\right. \\
= & \frac{\left(4 \lambda \mu C_{1}-a^{2} C^{2}\right)}{\left[(\mu k+d)+a C \sqrt{\left\{\left(\frac{(\mu k-d)}{a C}\right)^{2}+\frac{1}{\lambda}\right\}}\right.} .
\end{aligned}
$$


The maximum value of $\left(C^{*}\right)^{-1}$ is not easily determined from an examination of its derivative. Instead, we introduce the positive constant $M_{4}$ that satisfies

$$
\frac{a^{2} C^{2}}{4 C_{1}}<M_{4} \leq \infty
$$

and set

$$
\lambda=M_{4}^{2}, \quad \mu=M_{4}^{-1}, \quad \lambda \mu=M_{4},
$$

to obtain from (A.3)

$$
\begin{aligned}
\left(C^{*}\right)^{-1}= & \frac{1}{\left(4 M_{4} k d-a^{2} C^{2}\right)}\left[\left(M_{4}+C_{1}\right)-\sqrt{\left(\left(M_{4}-C_{1}\right)^{2}+a^{2} C^{2}\right)}\right] \\
& \times\left[\left(k+M_{4} d\right)-\sqrt{\left(\left(k-M_{4} d\right)^{2}+a^{2} C^{2}\right)}\right]
\end{aligned}
$$

We now select

$$
M_{4}=\frac{a^{2} C^{2}}{4 k d p},
$$

where $p>0$. In order to satisfy (5.9) and (A.4), we require

$$
0<p<\frac{(1-k e C)}{k d}<(k d)^{-1}<1,
$$

the last inequality being derived from the properties $k>1, d \geq 1$, which are valid by definition of $k$ and $d$. Substitution of (A.7) in (A.6) leads to corresponding rates of growth and decay as previously explained. Note that $p$ cannot be arbitrarily small (and therefore $M_{4}$ arbirtrarily large) since (A.5) implies that then $\lambda \rightarrow \infty$ and $\mu \rightarrow 0$, while $\lambda \mu \rightarrow \infty$, and the definitions of $\Lambda(z)$ and $F(z)$ are invalidated.

\section{A.2 Method 2}

Indicative rates may be provided for certain geometries by taking $\gamma_{2}=\overline{\gamma_{2}}$, defined in (6.16), and $\gamma_{1}$ to be the mean value of the range (5.8); that is, we insert

$$
\gamma_{1}=\frac{\left(a^{2} C^{2}+4 \lambda \mu C_{1}\right)}{4 a C}, \quad \gamma_{2}=\overline{\gamma_{2}},
$$


into (6.21) to obtain the rate of growth and decay proportional to $\left(C^{*}\right)^{-1}$, where routine calculations show that

$$
\begin{aligned}
Y_{1}= & \frac{\left(4 \lambda \mu C_{1}-a^{2} C^{2}\right)}{\left(4 \lambda \mu C_{1}+a^{2} C^{2}\right)}, \\
Y_{3}= & \frac{\left(4 \lambda \mu C_{1}-a^{2} C^{2}\right)}{8}, \\
Y_{2}= & \frac{\left(4 \lambda \mu C_{1}-a^{2} C^{2}\right)}{8\left(4 \lambda \mu C_{1}+a^{2} C^{2}\right)}\left[4\left(2 k-d C_{1}\right)-d a^{2} C^{2}\right], \\
Y_{4}^{2}= & \left(\frac{\left(4 \lambda \mu C_{1}-a^{2} C^{2}\right)}{2 \sqrt{2}\left(4 \lambda \mu C_{1}+a^{2} C^{2}\right)}\right)^{2} \\
& \times\left[16\left(2 k-d C_{1}\right)^{2}-4 a^{2} C^{2}\left\{2 d\left(2 k-d C_{1}\right)-\lambda \mu C_{1}\right\} a^{4} C^{4}\left(1+d^{2}\right)\right], \\
& \frac{\left(4 \lambda \mu C_{1}-a^{2} C^{2}\right)}{\left(4 \lambda \mu C_{1}+a^{2} C^{2}\right)}\left[a^{2} C^{2}\right. \\
& +2 d\left[16\left(2 k-d C_{1}\right)^{2}-4 a^{2} C^{2}\left\{2 d\left(2 k-d C_{1}\right)-\lambda \mu C_{1}\right\} a^{4} C^{4}\left(1+d^{2}\right)\right]^{1 / 2} \\
& \left.+d a^{2} C^{2}-4\left(2 k-d C_{1}\right)\right] .
\end{aligned}
$$

Now suppose that

$$
0<2 k-d C_{1} \equiv k(2+d e C)-d,
$$

which is satisfied for sufficiently large $e$, or sufficiently small $h$, and any given $C$. Next, set

$$
\lambda \mu=\frac{2 d\left(2 k-d C_{1}\right)}{C_{1}},
$$

which from (5.9) requires that

$$
a^{2} C^{2}<d[k(2+d e C)-d]
$$

which always holds provided $C$ lies in the range

$$
e d^{2}-\sqrt{\left(e^{2} d^{4}-4 a^{2} d(d-2 k)\right)} \leq C \leq e d^{2}+\sqrt{\left(e^{2} d^{4}-4 a^{2} d(d-2 k)\right)}
$$

and

$$
4 a^{2}(d-2 k) \leq e^{2} d^{3} .
$$

The last condition is valid for sufficiently large $e$.

Then we have

$$
\left(C^{*}\right)^{-1}=Y_{1} \frac{\left[4(2 \sqrt{2}-1)\left(2 k-d C_{1}\right)+d a^{2} C^{2}\right]}{4\left[4(2 d-1)\left(2 k-d C_{1}\right)+a^{2} C^{2}(1+d)\right]},
$$

where

$$
Y_{1}=\frac{\left[2 d\left(2 k-d C_{1}\right)-a^{2} C^{2}\right]}{\left[2 d\left(2 k-d C_{1}\right)+a^{2} C^{2}\right]},
$$

and is positive by virtue of assumption (A.10).

Subject to the above stated conditions, growth and decay rates now follow as described previously. 


\section{A.3 Method 3}

A third possible choice of constants consists in putting

$$
\gamma_{1}=\frac{b C_{1}^{1 / 2}}{C_{2}}
$$

for arbitrary positive constant $C_{2}$. The estimate (4.8) is replaced by

$$
\left|\int_{D(z)} \beta_{i j} u_{i, j} \theta d a\right| \leq C_{2}\left[\int_{D(z)} d_{i j k h} u_{i, j} u_{k, h} d a+C_{1} \Lambda_{0}(z) \int_{D(z)} k_{i j} \theta_{, i} \theta_{, j} d a\right],
$$

where

$$
\begin{aligned}
\Lambda_{0}(z) & =\frac{b^{2}}{C_{2}^{2}}(z+h)^{2 \alpha} \\
& =\frac{b^{2}}{\lambda C_{2}^{2}} \Lambda(z) .
\end{aligned}
$$

Consequently, Lemma 2 holds for arbitrarily large positive constant $C_{2}$. We restrict, however, the constant to satisfy $0<C_{2}<1$. It will become apparent in what follows that while the choice (A.13) leads to certain simplications, it presents difficulties when determining optimum growth and decay rates.

To proceed, we further set

$$
\lambda \mu=\left(\frac{b}{C_{2}}\right)^{2},
$$

which in conjunction with (5.9) implies that $C_{2}^{2}<1$, while definitions (5.6) and (5.7) reduce to

$$
\begin{aligned}
A & =\left(1-C_{2}\right) \\
B & =\mu C_{1}-\frac{a b C C_{1}^{1 / 2}}{2 \lambda C_{2}} \\
& =\mu C_{1}-\frac{b^{2} C_{1}}{\lambda C_{2}} \\
& =\left(\frac{b}{C_{2}}\right)^{2} \frac{C_{1}}{\lambda}\left(\lambda \mu\left(\frac{C_{2}}{b}\right)^{2}-C_{2}\right) \\
& =\left(\frac{b}{C_{2}}\right)^{2} \frac{C_{1}}{\lambda}\left(1-C_{2}\right) .
\end{aligned}
$$

It follows from (A.13) and (A.14) that

$$
B \Lambda(z)=C_{1}\left(1-C_{2}\right) \Lambda_{0}(z)
$$

and that the lower bound (5.5) becomes

$$
F^{\prime}(z) \geq\left(1-C_{2}\right)\left[\int_{D(z)} d_{i j k l} u_{i, j} u_{k, l} d a+C_{1} \Lambda_{0}(z) \int_{D(z)} k_{i j} \theta_{, i} \theta_{, j} d a\right] .
$$


For the particular choice of $\gamma_{2}$, we set

$$
\gamma_{2}=\frac{a C}{2 C_{2}},
$$

Subject to the further special choices (A.13) and (A.15), we then have

$$
\begin{aligned}
U & =\left(d+C_{2}\right) \\
V & =\frac{b^{2} C_{1}}{\lambda C_{2}^{2}}\left(C_{2}+k C_{1}^{-1}\right) \\
& =\frac{1}{\lambda}\left(\frac{a c}{2 C_{2}}\right)^{2}\left(C_{2}+k C_{1}^{-1}\right) .
\end{aligned}
$$

On substituting from (A.16), (A.17), (A.21), and (A.22) in (5.4) and (5.11) we conclude that

$$
\begin{aligned}
|F(z)| \leq & C(z+h)^{\alpha}\left[\left(d+C_{2}\right) \int_{D(z)} d_{i j k l} u_{i, j} u_{k, l} d a\right. \\
& \left.+\frac{b^{2} C_{1}}{\lambda C_{2}^{2}} \Lambda(z)\left(C_{2}+k C_{1}^{-1}\right) \int_{D(z)} k_{i j} \theta_{, i} \theta_{, j} d a\right] \\
\leq & n C(z+h)^{\alpha}\left[\int_{D(z)} d_{i j k l} u_{i, j} u_{k, l} d a+\frac{b^{2} C_{1}}{\lambda C_{2}^{2}} \Lambda(z) \int_{D(z)} k_{i j} \theta_{, i} \theta_{, j} d a\right] \\
= & n C(z+h)^{\alpha}\left[\int_{D(z)} d_{i j k l} u_{i, j} u_{k, l} d a+C_{1} \Lambda_{0}(z) \int_{D(z)} k_{i j} \theta_{, i} \theta_{, j} d a\right] \\
\leq & \frac{n C}{\left(1-C_{2}\right)}(z+h)^{\alpha} F^{\prime}(z) \\
= & C^{*} C(z+h)^{\alpha} F^{\prime}(z),
\end{aligned}
$$

where we have used (A.14), and set

$$
\begin{aligned}
n & =\max \left\{\left(C_{2}+d\right),\left(C_{2}+k C_{1}^{-1}\right)\right\}, \\
C^{*} & =n\left(1-C_{2}\right)^{-1} .
\end{aligned}
$$

The differential inequality (A.25) is consistent with the generic form whose integration has been previously discussed.

It has already been remarked that optimum growth and decay rates are achieved for minimum values of the generic constant $C^{*}$. That is, for the present special choice of $\gamma_{1}, \gamma_{2}$, we wish to choose $C_{2}$ to ensure that

$$
\left(C^{*}\right)^{-1}=\frac{\left(1-C_{2}\right)}{\left(C_{2}+q\right)}
$$

is a maximum, where

$$
q=\max \left(d, k C_{1}^{-1}\right) .
$$


It follows in these circumstances that the optimum value $q$ of $C^{*}$, attained in the limit $C_{2} \rightarrow 0$, is not achievable because several quantities entering into the above calculations become unbounded rendering the conclusions meaningless. In particular, note that from (A.15), in the limit we also have $\lambda \mu \rightarrow \infty$, so that either $\lambda$ or $\mu$ or both must be infinite invalidating the choice of $\Lambda(z)$ or $F(z)$ or both.

Of course, explicit rates are obtained upon selecting a definite value for $C_{2}$ in the interval $0<C_{2}<1$, say $C_{2}=0.25$. Details are omitted. 